\title{
A Millimeter-Wave Second-Order All-Pass Delay Network in BiCMOS
}

\author{
Piotr Jan Osuch, Member, IEEE and Tinus Stander, Senior Member, IEEE
}

\begin{abstract}
Analogue signal processing (ASP) is a promising alternative to DSP techniques in $\mathrm{mm}$-wave technologies such as 5G, with second-order all-pass networks a key building block in ASPs. We present an active on-chip mm-wave second-order allpass network in a $130 \mathrm{~nm} 280 \mathrm{GHz} f_{\max } \mathrm{SiGe} B$ BiCMOS process with an effective bandwidth of $40 \mathrm{GHz}$, peak delay of 62 ps at $36 \mathrm{GHz}$, delay $Q_{D}$-value of 3.6 and a magnitude ripple of $1.4 \mathrm{~dB}$. A layoutfocused design methodology incorporating layout parasitics and process tolerances is followed. This is the first reported mm-wave bandwidth second-order all-pass network, and the first monolithic microwave integrated all-pass network with a $Q_{D}$-value greater than 1.
\end{abstract}

Index Terms-All-pass networks, analogue signal processing, group-delay engineering, millimeter wave integrated circuits, ultra-wideband technology.

\section{INTRODUCTION}

$\mathrm{D}$ ATA traffic of future $5 \mathrm{G}$ telecommunication systems is anticipated to increase 10000 -fold compared to current rates, necessitating wideband mm-wave front-haul links [1]. One possible mitigating approach to processing wideband data in real-time is to replace some baseband DSP blocks with ASP equivalents at RF [2].

Two fundamental building blocks of ASPs are dispersive first- and second-order all-pass networks [3] (as opposed to flat delay used in true time delay networks [4], [5]) for which both passive and active implementations have been proposed [6][11]. In many applications, a large band-pass delay $Q$-value $\left(Q_{D}\right)$ [2], [6] is required. Real-time spectrum analysis [6] requires $Q_{D}>0.79$ for frequency discrimination, while $Q_{D}>3.5$ and $Q_{D}>10$ results in a resolution of $\sim 0.4 f_{0}$ and $\sim 0.2 f_{0}$, respectively. In frequency scanning antenna arrays, a $Q_{D}$ of 2 has been shown to result in a mapping of $60^{\circ} / \mathrm{GHz}$ [6], with higher $Q_{D}$ required for finer spatial resolution. In M-ary pulseposition modulation (PPM), a $Q_{D}$ of 3.14 is required to create a maximum delay of one pulse width. Increasing $Q_{D}$ however, also increases the insertion loss of the network at resonance, which is exacerbated by low attainable on-chip inductor $Q$ factors (typically less than 10) [11]. In response to this, active implementations of all-pass networks have been proposed [7][11], but none achieve $Q_{D}$-values larger than 0.2 .

Manuscript received 26 February 2018, revised 28 May 2018. The financial assistance of the South African SKA Project (SKA SA - www.ska.ac.za), the Eskom Tertiary Education Support Programme (TESP), the National Research Foundation of South Africa (Grant 93921) and the MOSIS Educational Programme towards this research is hereby acknowledged.
A suitable single transistor second-order all-pass network with inductor $Q$-factor enhancement was proposed in [12], but the published synthesis method relies on zero length interconnects and ideal components. In MMICs, the circuit's design would be complicated by layout RLC parasitics and finite component Q-factors.

In this work, we present an MMIC second-order all-pass response with a $Q_{D}$-value larger than 1 for the first time, through augmentation of the procedure in [12] with an optimizationbased, layout-focused design methodology which incorporates accurate device models as well as layout-specific RC parasitic extraction. The effects of component tolerances on the group delay and magnitude responses are further investigated. The proposed design is prototyped in a $130 \mathrm{~nm}$ SiGe BiCMOS process as proof of concept.

\section{Design Procedure AND Simulation}

The single-transistor second-order all-pass network (based on [12]) is shown in Fig. 1. A single HBT npn transistor $\left(L_{e}=6\right.$ $\mu \mathrm{m}, W_{e}=120 \mathrm{~nm}$, multiplicity $\left.=4\right)$ is used with emitter parameters as shown. Transmission lines $T_{1}$ and $T_{2}$, each placed over a deep trench, act as inductors connected in series with capacitor $C_{l}$ to form a resonant $\mathrm{LC}$ tank. Interconnects over the shielding ground plane are explicitly modelled from the foundry PDK pcells as inductive elements $T_{3}$ and $T_{4}$. This approach models potential distributed inductance in long interconnects with foundry-qualified PDK pcells, negating the need for FEM modelling of the layout. The $V_{D D}$ bias connection is modelled as a $5 \mathrm{nH}$ inductor, though the circuit's performance is found largely insensitive to this choice. A decoupling capacitor is included as $C_{3}$, with a gain-enhancement capacitor $\mathrm{C}_{2}$ added to extend the bandwidth of the network. Further RC layout parasitics (as opposed to device parasitics and inductive interconnects included in foundry PDK pcell models) are further extracted and included in the final layout-ready simulation as demonstrated later.

The following design procedure is followed:

(i) Design equations from [12] are used for initial component values neglecting layout parasitics $\left(C_{3} \rightarrow \infty, C_{2} \rightarrow 0\right)$

(ii) An optimization is performed in simulation using the foundry PDK and initial values. $C_{3}$ is minimized without compromising decoupling, while $C_{2}$ is optimized to reduce high-frequency peaking.

P. J. Osuch and T. Stander are with the Carl and Emily Fuchs Institute for Microelectronics, Department of Electrical, Electronic and Computer Engineering, University of Pretoria, Pretoria, South Africa (e-mail Piotr.Osuch@up.ac.za, Tinus.Stander@up.ac.za).

Digital Object Identifier:10.1109/LMWC.2018.2863215

(c) 2018 IEEE. Personal use of this material is permitted. Permission from IEEE must be obtained for all other users, including reprinting/ republishing this material for advertising or promotional purposes, creating new collective works for resale or redistribution to servers or lists, or reuse of any copyrighted components of this work in other works 


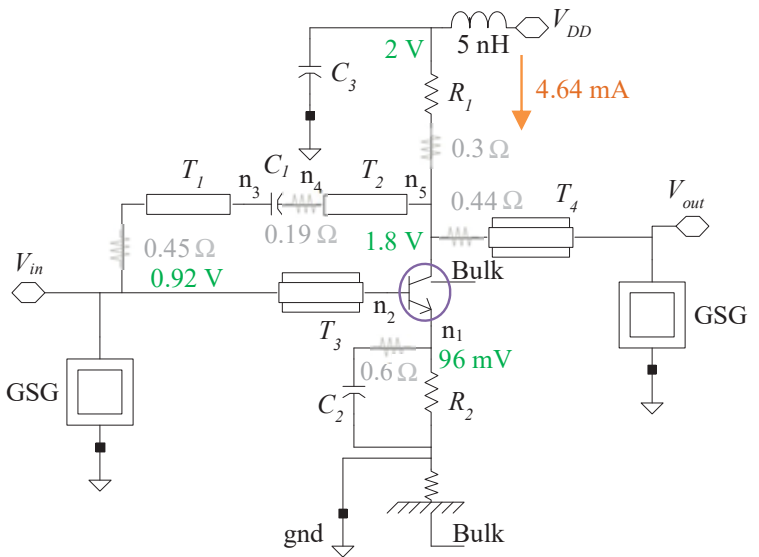

Fig. 1. Schematic of the single-transistor second-order all-pass network Parasitic resistances extracted from the final layout are shown.

(iii) Using the values obtained in (ii), a layout is constructed. $\mathrm{RC}$ layout parasitics are extracted with an automated tool, while inductive parasitics $T_{3}$ and $T_{4}$ are explicitly included in the schematic from pcells.

(iv) A second optimization is performed, this time including all parasitics $\left(T_{3}, T_{4}\right.$, and extracted $\left.\mathrm{RC}\right)$.

(v) The layout is updated to reflect the optimized changes.

\section{A. Theoretical calculations}

The ideal second-order all-pass voltage transfer function of Fig. 1. can be written as:

$$
T(s)=K \cdot \frac{s_{n}{ }^{2}-s_{n} / Q_{D}+1}{s_{n}{ }^{2}+s_{n} / Q_{D}+1},
$$

where $s_{n}=s / \omega_{0}, Q_{D}=\Delta \tau \omega_{0} / 4$ is the delay $Q$-value, $\omega_{0}=$ $2 \pi f_{0}$ is the center frequency of the second-order all-pass delay function and $\Delta \tau$ is the corresponding peak-to-nominal group delay. Equating similar terms in (2) with those in [12] leads to:

$$
\begin{gathered}
\frac{1}{\omega_{0}^{2}}=L C_{1}, \\
\frac{1}{Q \cdot \omega_{0}}=\frac{C_{1}}{G_{2}-G},
\end{gathered}
$$

where $L$ is the combined inductance of $T_{1}$ and $T_{2}, G_{2}=1 / R_{2}$, and $G$ is the combined parasitic conductance in parallel with $T_{1}$, $T_{2}$ and $C_{l}$. Conductance $G_{1}$ is chosen as detailed in [12] to meet the all-pass condition.

Applying step (i) of the design procedure using (1) - (3) and [12], we set $\Delta \tau=38 \mathrm{ps}, f_{0}=30 \mathrm{GHz}, G=0.04 \mathrm{~S}, G_{2}=0.086$ $\mathrm{S}$. As the proposed network is designed to operate as a voltagemode device, $50 \Omega$ impedance matching and return loss are not considered as goals. This results in in $G_{1}=0.025 \mathrm{~S}, C_{1}=136$ $\mathrm{fF}$ and $L=207 \mathrm{pH}$. Initial values for $C_{2}$ and $C_{3}$ are chosen as $60 \mathrm{fF}$ and $75 \mathrm{pF}$ respectively. Using these values, the circuit schematic of Fig. 1 (excluding layout parasitics and interconnects) is simulated using the foundry PDK. The results are shown in Fig. 2 (blue trace). The effect of non-idealities is clear from the initial transmission magnitude variation of $7 \mathrm{~dB}$ and delay peak offset of $4 \mathrm{GHz}$.

\section{B. Application of layout-based optimization}

The first optimization is now performed to account for the device non-idealities (step (ii) of the procedure), where $f_{0}, \Delta \tau$ and passband magnitude variation are set as gradient-based optimization goals with $C_{1}, C_{2}, L, R_{1}$ and $R_{2}$ chosen as variables. The resulting magnitude and group delay responses in Fig. 2 (red trace) indicate that the passband magnitude variation is improved to $0.8 \mathrm{~dB}$, with $f_{0}$ and $\Delta \tau$ goals met.
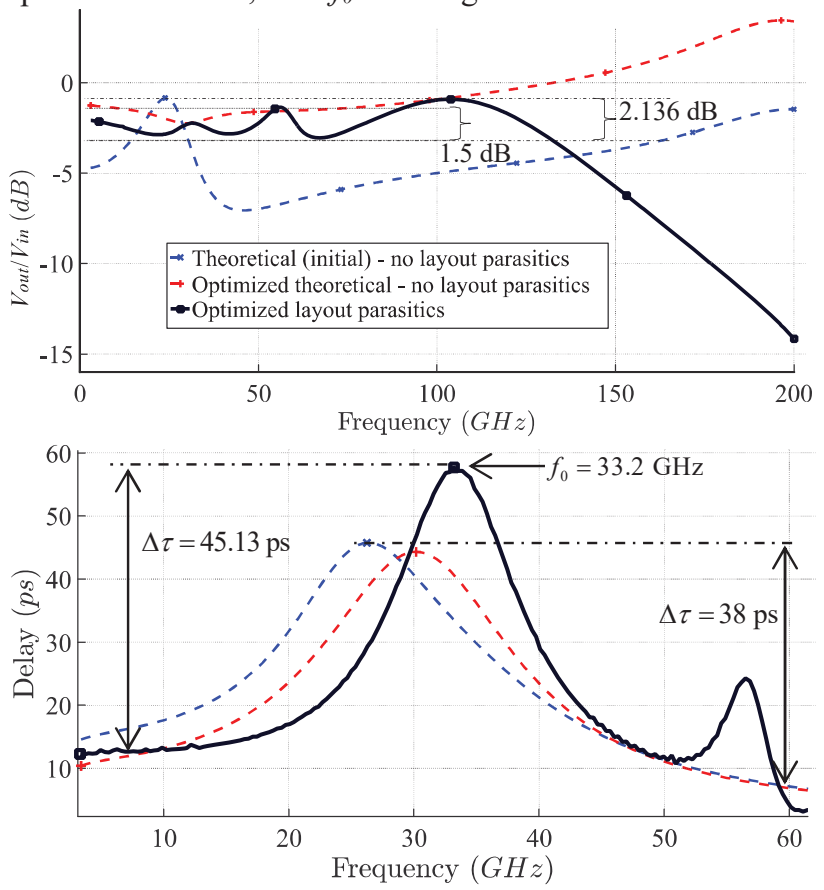

Fig. 2. Transmission magnitude and delay responses (voltage transfer).

Next, in step (iii), a layout is drawn as shown in Fig. 3 and layout parasitics extracted. $T_{3}$ and $T_{4}$ are included explicitly, as these were assumed zero in step (ii).

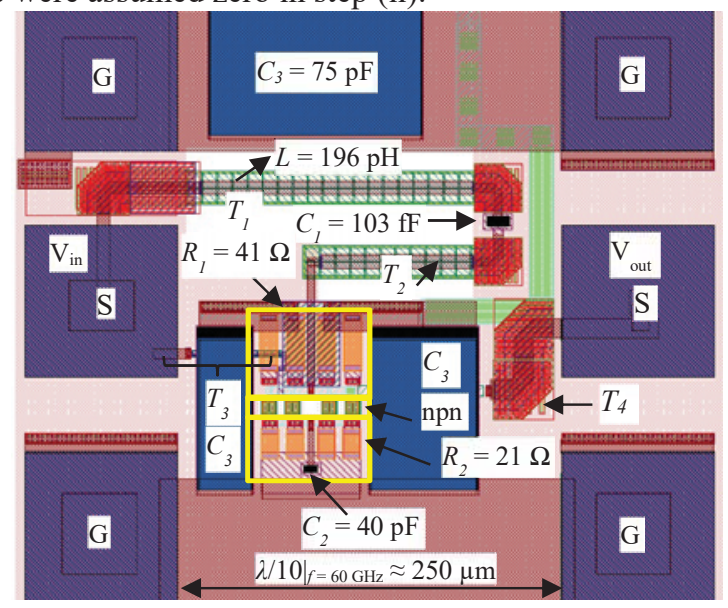

Fig. 3. Camera-ready layout of the BiCMOS second-order all-pass network.

146 parasitic capacitors and 35 parasitic resistors are extracted from the layout, with the dominant layout parasitic resistors indicated in Fig. 1. The sum of layout parasitic capacitances at each node are comparable to some component values (e.g. $C_{1}$ ) justifying the RC extraction and layout-based approach. Another optimization is performed (step (iv)) using the same optimization goals and variables, but now including the extracted $\mathrm{RC}$ layout parasitics. The resulting responses are shown in Fig. 2 (black trace). 
It is found that the initial optimization parameters do not offer sufficient variation to meet all the pre-defined goals whilst maintaining pass-band variation of below $1.5 \mathrm{~dB}$. This is a known trade-off in designing high- $Q_{D}$ networks [2], [3]. The final achieved values of $f_{0}=33.2 \mathrm{GHz}$ and $\Delta \tau=45.13 \mathrm{ps}$ with magnitude variation of $1.5 \mathrm{~dB}$ (2.14 $\mathrm{dB}$ including peaking) represent a fair trade-off between passband flatness and deviation from desired $\Delta \tau$ and $f_{0}$. The network's bandwidth is limited to $50 \mathrm{GHz}$ by a spurious second delay peak at $56 \mathrm{GHz}$. An average output noise of $0.69 \mathrm{nV} / \sqrt{\mathrm{Hz}}$ is simulated over the band.

\section{MEASUREMENT RESUltS}

The design is prototyped in the GlobalFoundries US 8HP 130 $\mathrm{nm}$ SiGe BiCMOS process, and measured with $150 \mu \mathrm{m}$ pitch GSG wafer probes (Fig. 4, insert) on an Anritsu ME7828A VNA, with a single DC bias needle supplying $V_{D D}$. The network consumes $9.3 \mathrm{~mW}$. The measured S-parameters are converted to $\mathrm{ABCD}$ parameters and the voltage transfer is extracted as shown in Fig. 4, with substantial dispersion observable down to $\sim 20 \mathrm{GHz}$. A magnitude response ripple of $1.4 \mathrm{~dB}$ over a bandwidth of $40 \mathrm{GHz}$ is measured. The obtained $\Delta \tau$ of $62.4 \mathrm{ps}$ is larger than the simulated value of $45 \mathrm{ps}$, and $f_{0}$ is offset by 6.23 GHz. The poor absolute tolerances of on-chip TaN resistors (also shown in Fig. 4) may partially explain the magnitude ripple and $\Delta \tau$ discrepancies, but Fig. 2 would indicate that discrepancy in $f_{0}$, is, more probably, the result of unmodeled parasitics.
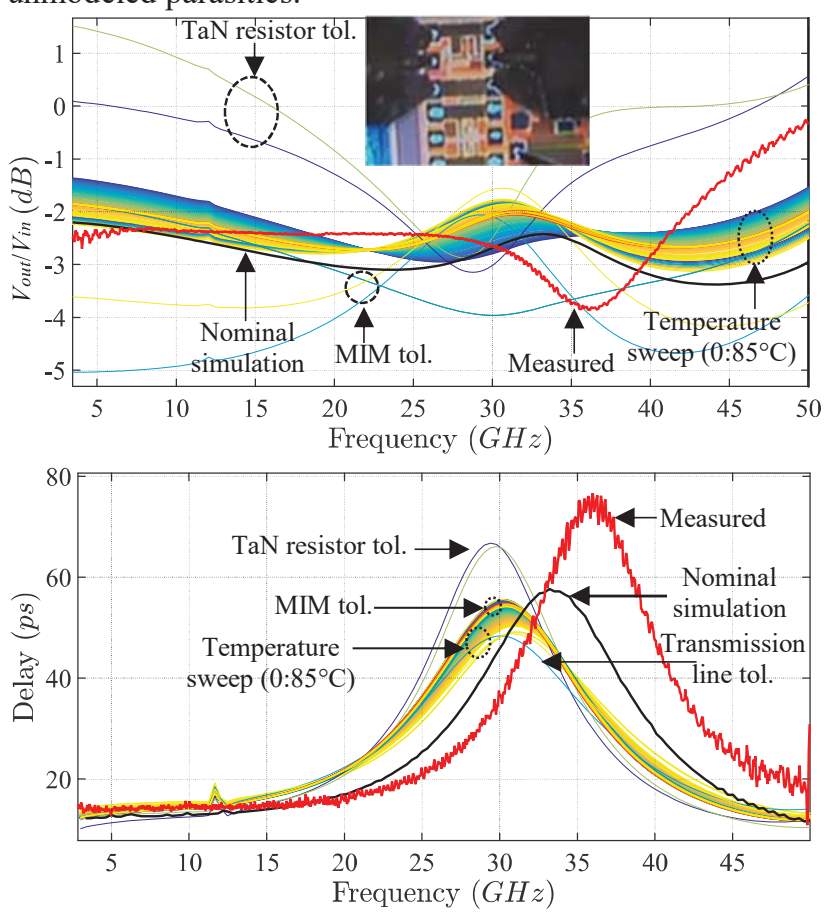

Fig. 4. Measured magnitude and delay responses (voltage transfer).

$\mathrm{P} 1 \mathrm{db}$ is measured at $36 \mathrm{GHz}$ as $-6.7 \mathrm{dBm}$. The performance is compared to other state-of-the-art designs in Table I.
TABLE I

Comparison with measured $2^{\text {nd }}$ order networks above $4 \mathrm{GHz}$.

\begin{tabular}{llllllll}
\hline \hline & $Q_{D}$ & $\begin{array}{l}f_{0} \\
(\mathrm{GHz})\end{array}$ & $\begin{array}{l}-3 \mathrm{~dB} \\
(\mathrm{GHz})\end{array}$ & Technology & $\begin{array}{l}\text { Size } \\
\left(\mathrm{mm}^{2}\right)\end{array}$ & $\begin{array}{l}\text { Power } \\
(\mathrm{mW})\end{array}$ & $\begin{array}{l}\Delta|T| \\
(\mathrm{dB})^{* *}\end{array}$ \\
\hline$[*]$ & 3.6 & 36 & 40 & $0.13 \mu \mathrm{m} \mathrm{SiGe}$ & 0.0625 & 9.3 & 1.4 \\
{$[4]$} & 0 & 0 & 12.2 & $0.16 \mu \mathrm{m}$ CMOS & 0.07 & 90 & $\sim 1.4$ \\
{$[5]$} & 0 & 0 & 4.38 & $0.18 \mu \mathrm{m} \mathrm{CMOS}$ & 0.0512 & 7.88 & - \\
{$[7]$} & 0.19 & 3 & 4 & $0.25 \mu \mathrm{m} \mathrm{CMOS}$ & 0.085 & $<95$ & $\sim 1.5$ \\
{$[8]$} & 0.04 & 7 & 13 & $0.13 \mu \mathrm{m} \mathrm{CMOS}$ & 0.0627 & 18.5 & $\sim 0.5$ \\
{$[9]$} & 0.098 & 7 & 16.5 & $0.09 \mu \mathrm{m} \mathrm{CMOS}$ & - & $<27$ & $<1$ \\
{$[10]$} & 0.049 & 6.3 & 12 & $0.13 \mu \mathrm{m} \mathrm{CMOS}$ & - & 16.5 & $\sim 1.5$ \\
{$[11]$} & 0.047 & 6 & 7.5 & $0.25 \mu \mathrm{m} \mathrm{SiGe}$ & $0.49^{\#}$ & 121 & $\sim 1$ \\
\hline${ }^{*} \mathrm{~N}$
\end{tabular}

first-order sections (no complex pole/zero).

${ }^{\#}$ Including pads. ${ }^{* *} \mathrm{~T}$ represents either a power or voltage transfer function.

\section{CONCLUSION}

An optimization-based, layout-focused design procedure is applied to produce the first dispersive active on-chip mm-wave second-order all-pass network with a delay $Q_{D}$-value of 3.6, which enables various ASP applications for the first time. Future work will focus on synthesizing higher-order delay functions by cascading multiple second-order all-pass sections, introducing post-production tuning mechanisms for $f_{0}$ and $\Delta \tau$, and including impedance matching for $50 \Omega$ system integration.

\section{REFERENCES}

[1] A. Ghosh et al., "Millimeter-wave enhanced local area systems: a highdata-rate approach for future wireless networks," IEEE Journal on Selected Areas in Communications, vol. 32, no. 6, pp. 1152-1163, 2014.

[2] S. Gupta, A. Parsa, E. Perret, R. V. Snyder, R. J. Wenzel, and C. Caloz, "Group-delay engineered noncommensurate transmission line all-pass network for analog signal processing," IEEE Transactions on Microwave Theory and Techniques, vol. 58, no. 9, pp. 2392-2407, 2010.

[3] P. J. Osuch and T. Stander, "A geometric approach to group delay network synthesis," Radioengineering, vol. 25, no. 2, pp. 351-364, 2016.

[4] S. K. Garakoui, E. A. Klumperink, B. Nauta, and F. E. van Vliet, "Compact cascadable gm-C all-pass true time delay cell with reduced delay variation over frequency," IEEE Journal of Solid-State Circuits, vol. 50, no. 3, pp. 693-703, 2015.

[5] Y. W. Chang, T. C. Yan, and C. N. Kuo, "Wideband time-delay circuit," in European Conference In Microwave Integrated Circuits, 2011, pp. 454-457.

[6] C. Caloz, S. Gupta, Q. Zhang, and B. Nikfal, "Analog signal processing: a possible alternative or complement to dominantly digital radio schemes," IEEE Microwave Magazine, vol. 14, no. 6, pp. 87-103, 2013.

[7] X. Lin, J. Liu, H. Lee, and H. Liu, "A 2.5- to 3.5-Gb/s adaptive FIR equalizer with continuous-time wide-bandwidth delay line in $0.25-\mu \mathrm{m}$ CMOS," IEEE Journal of Solid-State Circuits, vol. 41, no. 8, pp. 19081918, 2006.

[8] P. Ahmadi, B. Maundy, A. S. Elwakil, L. Belostotski, and A. Madanayake, "A new second-order all-pass filter in 130-nm CMOS," IEEE Transactions on Circuits and Systems II: Express Briefs, vol. 63, no. 3, pp. 249-253, 2016.

[9] M. Maeng et al., "0.18-um CMOS equalization techniques for $10-\mathrm{Gb} / \mathrm{s}$ fiber optical communication links," IEEE Transactions on Microwave Theory and Techniques, vol. 53, no. 11, pp. 3509-3519, 2005.

[10] P. Ahmadi, M. H. Taghavi, L. Belostotski, and A. Madanayake, "10-GHz current-mode 1 st and 2 nd order allpass filters on 130nm CMOS," in IEEE 56th International Midwest Symposium on Circuits and Systems, 2013, pp. $1-4$.

[11] M. Hamouda, G. Fischer, R. Weigel, and T. Ussmueller, "A compact analog active time delay line using SiGe BiCMOS technology," in 2013 IEEE International Symposium on Circuits and Systems, 2013, pp. 10551058 .

[12] H. Rubin and R. Even, "Single-transistor all-pass networks," IEEE Transactions on Circuit Theory, vol. 20, no. 1, pp. 24-30, 1973. 